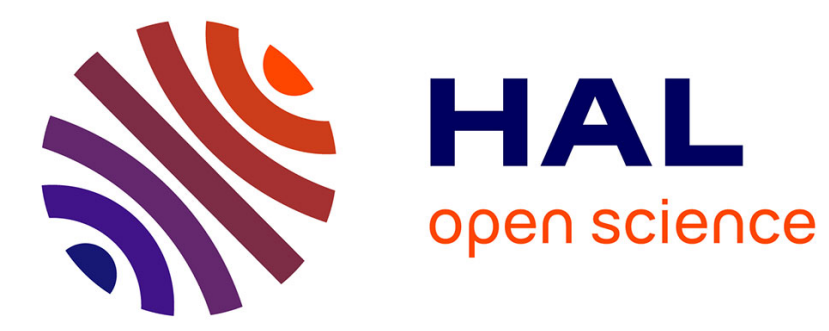

\title{
A variational Bayesian approximation approach via a sparsity enforcing prior in acoustic imaging
}

Ning Chu, Ali Mohammad-Djafari, Nicolas Gac, José Picheral

\section{To cite this version:}

Ning Chu, Ali Mohammad-Djafari, Nicolas Gac, José Picheral. A variational Bayesian approximation approach via a sparsity enforcing prior in acoustic imaging. WIO 2014, Jul 2014, Neuchâtel, Switzerland. pp.1 - 4, 10.1109/WIO.2014.6933297 . hal-01103751

\section{HAL Id: hal-01103751 \\ https://hal-centralesupelec.archives-ouvertes.fr/hal-01103751}

Submitted on 15 Jan 2015

HAL is a multi-disciplinary open access archive for the deposit and dissemination of scientific research documents, whether they are published or not. The documents may come from teaching and research institutions in France or abroad, or from public or private research centers.
L'archive ouverte pluridisciplinaire HAL, est destinée au dépôt et à la diffusion de documents scientifiques de niveau recherche, publiés ou non, émanant des établissements d'enseignement et de recherche français ou étrangers, des laboratoires publics ou privés. 


\section{A Variational Bayesian Approximation Approach via a Sparsity Enforcing Prior in Acoustic Imaging}

\author{
Ning Chu, Ali Mohammad-Djafari, Nicolas Gac \\ Laboratoire des signaux et systèmes (L2S) \\ UMR 8506, CNRS-SUPELEC-UNIV PARIS SUD \\ 3 Rue Joliot-Curie, 91192 Gif-sur-yvette, France \\ Email: chuning1983@gmail.com,djafari@lss.supelec.fr
}

\author{
José Picheral \\ Dept. Signal et Systèmes Électroniques \\ École supérieure d'électricité (SUPELEC) \\ 3 Rue Joliot-Curie, 91192 Gif-sur-yvette, France
}

\begin{abstract}
Acoustic imaging is an advanced technique for acoustic source localization and power reconstruction from limited noisy measurements at microphone sensors. To solve this ill-posed inverse problem, the Bayesian inference methods using proper prior knowledge have been widely investigated. In this paper, we propose to use a hierarchical Variational Bayesian Approximation for the robust acoustic imaging. And we explore the Student's-t priors with heavy tails to enforce source sparsity and non-Gaussian noises, so that we can achieve the super spatial resolution and wide dynamic range of source powers. In addition, proposed approach is validated by simulations and real data from
\end{abstract} wind tunnel in automobile industry.

Keywords-Acoustic imaging; Variational Bayesian Approximation; Student's-t prior; non-Gaussian noises.

\section{INTRODUCTION}

In general, the conventional Beamforming [1] method can give a direct and fast acoustic power imaging, but its spatial resolution is often very coarse at low frequencies. Based on the beamforming, the forward model of acoustic power propagation can be modeled by a determined linear system of equations in frequency domain: $\mathbf{y}=\mathbf{C x}$, where $\mathbf{y}$ denotes observed beamforming power vector at microphone array; $\mathrm{x}$ denotes unknown acoustic power vector on the source plane; $\mathbf{C}$ denotes the propagation matrix, which usually depends on the geometric distance between the source and sensor for a given frequency. Therefore $\mathbf{C}$ is shift-variant and usually a singular matrix, which causes ill-poses problems. Recently, the Deconvolution Approach for Mapping of Acoustic Source (DAMAS) method [2] has effectively applied in wind tunnel experiments by the NASA. For super resolution imaging in strong Gaussian noises, many methods with sparsity constraints have been effectively developed [3], [4]. However, the sparsity parameters have to be selected empirically. In order to obtain the robust parameter estimations, we have recently the Bayesian inference approaches with the sparsity enforcing priors have been widely investigated [5], [6], [7], [8]. We have proposed to use the Double Exponential (DE) prior, and apply the Maximum A Posteriori (MAP) estimation [9] which gives better results than DAMAS and its extensions. However, the MAP method often confronts the time-consuming nonquadratic optimization.

In this paper, our motivation is to achieve the robust acoustic imaging on the vehicle surface in wind tunnel tests, which is widely used in car manufacture. To overcome the above difficulties, we propose to use a hierarchical Bayesian inference via Variational Bayesian Approximation (VBA). Moreover, the Student's-t prior is used to enforce the sparsity of source power distribution.

This paper is organized as follows: Section II introduces the forward model of acoustic power propagation; Proposed VBA approach is discussed in Section III; And method validations are carried out on simulations in Section IV and real data in Section V; Finally Section VI concludes this paper.

\section{Problem Statement}

We consider $\mathrm{M}$ sensors in a non-uniform 2D array and $\mathrm{K}$ unknown sources on the source plane. Some assumptions are made as: Acoustic sources are uncorrelated, monopoles [2]; Sensors are omni-directional with unitary gain. After discretization into $\mathrm{N}$ identical grids on the source plane, we get $\mathrm{N}$ potential sources with $K$ non-zero items (K-sparsity signal if $K<<N)$. Background noises are usually generated by both the noises at the sensors and the model uncertainty [9] caused by acoustic reverberation, reflection and refraction in wind tunnels. Thus background noises actually have colored frequency spectrum, instead of Gaussian white one in [2], [9]. Therefore we proposed the forward model of acoustic power propagation in colored noises as:

$$
\mathbf{y}=\mathbf{C x}+\boldsymbol{\xi}
$$

where $\boldsymbol{\xi}$ denotes the colored noises; Propagation matrix $\mathbf{C}=\left\{\left\|\mathbf{a}_{i}^{H} \mathbf{a}_{j}\right\|_{2}^{2} /\left\|\mathbf{a}_{i}\right\|_{2}^{2}\right\}_{N \times N}$ is obtained in [9], in which, the beamforming steering vector $\mathbf{a}_{i}=\left\{\frac{\exp \left[-j 2 \pi f r_{i, m}\right] / c_{0}}{r_{i, m}}\right\}_{M}$ [2] depends on the geometric distance $r_{i, m}$ between source $i$ and sensor $m$ at a given frequency $f$, with $c_{0}$ being acoustic speed in common air. Thus (1) is an under-determined linear system of equations for $\mathrm{x}$ and $\boldsymbol{\xi}$.

\section{PROPOSED VBA INFERENCE APPROACH}

For the inverse problem in (1), some prior information (constraints) on both source powers $\boldsymbol{x}$ and colored noises $\boldsymbol{\xi}$ should be investigated in order to reduce the uncertainty of solutions. Let $\boldsymbol{\theta}=[\boldsymbol{x}, \boldsymbol{\xi}]^{T}$ denote the unknown parameters, where operator $(\cdot)^{T}$ denotes transpose, and $\mathbf{D}$ denotes observed data and known parameters. The inverse problem via 
priors can be effectively solved by the following Bayesian inference approaches [5], [10], [6]: If we assign the specific prior probability $p(\boldsymbol{\xi})$ to noises $\boldsymbol{\xi}$, we can define the likelihood $p(\mathbf{D} \mid \boldsymbol{\theta})$ which is used classically by the Maximum Likelihood (ML) estimation as $\hat{\boldsymbol{\theta}}_{M L}=\arg \max _{\boldsymbol{\theta}}\{p(\mathbf{D} \mid \boldsymbol{\theta})\}$; In the Bayesian approach, we also assign the specific prior probabilities $p(\boldsymbol{\theta})$ to all unknown parameters. Then we use the MAP estimation as $\hat{\boldsymbol{\theta}}_{M A P}=\arg \max _{\boldsymbol{\theta}}\{\ln p(\boldsymbol{\theta} \mid \mathbf{D})\} \propto$ $\arg \min _{\boldsymbol{\theta}}\{-\ln p(\mathbf{D} \mid \boldsymbol{\theta})-\ln p(\boldsymbol{\theta})\}$ according to Baye's rule. In fact, MAP can be seen as a regularization of ML, but MAP has the advantage of adaptively estimating the regularization parameter, compared to conventional regularization methods. However in MAP, $\ln p(\boldsymbol{\theta} \mid \boldsymbol{D})$ is often hardly to get an analytical form and usually a nonlinear function with respect to $\boldsymbol{\theta}$. Moreover, both ML and MAP are the point estimators.

These difficulties can be overcome by the VBA [7], [8] estimation, in which, posterior $p(\boldsymbol{\theta} \mid \boldsymbol{D})$ is approximated by a family of basic easily handled probability distributions $q(\boldsymbol{\theta})$, namely $p(\boldsymbol{\theta} \mid \boldsymbol{D}) \approx q(\boldsymbol{\theta})$; and proper $q(\boldsymbol{\theta})$ are estimated by maximizing variational bound $\mathfrak{L}(\boldsymbol{\theta})$ as: $\hat{q}(\boldsymbol{\theta})=$ $\arg \max _{q(\boldsymbol{\theta})}\{\mathfrak{L}(\boldsymbol{\theta})\}$, where $\mathfrak{L}(\boldsymbol{\theta})=\int q(\boldsymbol{\theta}) \ln \frac{p(\boldsymbol{D}, \boldsymbol{\theta})}{q(\boldsymbol{\theta})} d \boldsymbol{\theta}$. Generally, $\boldsymbol{\theta}$ are supposed to be mutually independent: $q(\boldsymbol{\theta})=$ $\prod_{i} q\left(\theta_{i}\right)$. Then $\mathfrak{L}(\boldsymbol{\theta})$ is maximized by the mean field approximation as: $\hat{q}\left(\theta_{i}\right)=\frac{\exp \left[I\left(\theta_{i}\right)\right]}{\int \exp \left[I\left(\theta_{i}\right)\right] d \theta_{i}}$, where $I(\cdot)$ denotes the partition function, defined as $I\left(\theta_{i}\right)=<\ln p(\boldsymbol{D}, \boldsymbol{\theta})>_{q\left(\boldsymbol{\theta}_{-i}\right)}=$ $\int q\left(\boldsymbol{\theta}_{-i}\right) \ln p(\boldsymbol{D}, \boldsymbol{\theta}) d \boldsymbol{\theta}_{-i}$, where $\boldsymbol{\theta}_{-i}$ denotes the parameter vector except item $\theta_{i}$. In fact, $I\left(\theta_{i}\right)$ could hardly be analytically computed, since it depends on $q\left(\boldsymbol{\theta}_{-i}\right)$. But VBA inference can still obtain the approximating posterior $\hat{q}(\boldsymbol{\theta})$ owing to the conjugate distributions, in which, $\hat{q}(\boldsymbol{\theta})$ comes from the same family of the prior $p(\boldsymbol{\theta})$ based on the conjugate prior combination [11] of the likelihood and priors.

\section{A. Heavy tail prior on colored noises}

(a)

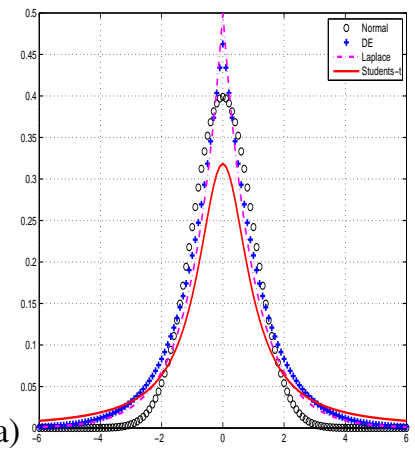

(b)

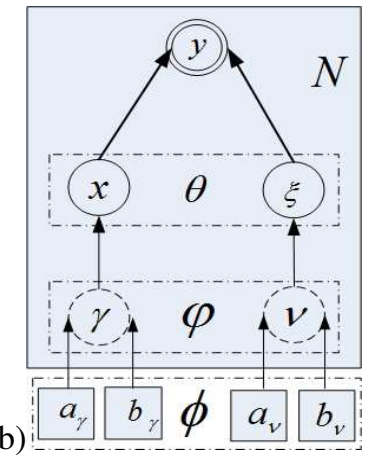

Fig. 1. (a) Sparse priors on Gaussian normal, Laplace, DE and Student's-t; (b) $N$ dimension hierarchical Bayesian Graphical model; Double circle: observed data; Single: unknown variables; Dash: hidden variables; Square: hyper-parameters; Arrow: dependence.

In wind tunnel experiments, we model the colored noises $\boldsymbol{\xi}$ by the Student's-t prior $\operatorname{St}(\boldsymbol{\xi})$ that has a long heavy tail, instead of Gaussian white ones whose sharp tail excessively penalizes the large errors of forward model. Another attractive superposition property is that $S t(\boldsymbol{\xi})$ can be generated by marginalizing hidden variable $\boldsymbol{\nu}$ as $S t(\boldsymbol{\xi})=$ $\int p(\boldsymbol{\xi} \mid \boldsymbol{\nu}) p(\boldsymbol{\nu}) d \boldsymbol{\nu}$, in which, conditional prior $p(\boldsymbol{\xi} \mid \boldsymbol{\nu})=$ $\mathcal{N}\left(\boldsymbol{\xi} \mid 0, \boldsymbol{\Sigma}_{\xi}^{-1}\right)$ is the multivariate Gaussian distribution, with $\boldsymbol{\Sigma}_{\xi}=\operatorname{Diag}\{\boldsymbol{\nu}\}$ being noise covariance matrix; $\operatorname{Diag}(\cdot)$ denotes diagonal matrix; $\boldsymbol{\nu}=\left\{\nu_{n}\right\}_{N}$ denotes the noise precision vector; And $p(\boldsymbol{\nu})=\prod_{n=1}^{N} \operatorname{Gama}\left(\nu_{n} \mid a_{\nu}, b_{\boldsymbol{\nu}}\right)=$ $\prod_{n=1}^{N} \Gamma\left(a_{\nu}\right)^{-1}\left(b_{\nu}\right)^{a_{\nu}} \nu_{n}^{a_{\nu}-1} e^{-b_{\nu} \nu_{n}}$, with $a_{\nu}, b_{\nu}$ being the hyperparameters of $p(\boldsymbol{\nu})$ and $\Gamma(x)=\int t^{x-1} e^{-t} d t$.

According to proposed forward model of (1), the likelihood is determined by the conditional prior $p(\boldsymbol{\xi} \mid \boldsymbol{\nu})=\mathcal{N}\left(\boldsymbol{\xi} \mid 0, \boldsymbol{\Sigma}_{\xi}^{-1}\right)$ as:

$$
p(\mathbf{y} \mid \mathbf{x}, \boldsymbol{\nu})=\frac{\left|\boldsymbol{\Sigma}_{\xi}\right|^{1 / 2}}{(2 \pi)^{N / 2}} e^{-\frac{1}{2}(\mathbf{y}-\mathbf{C x})^{H} \boldsymbol{\Sigma}_{\xi}(\mathbf{y}-\mathbf{C x})},
$$

where operator $(\cdot)^{H}$ denotes conjugate transpose.

\section{B. Sparse prior on acoustic power image}

Acoustic source in wind tunnel experiments are generated by the wind collision on the specific parts of the vehicle surface. Therefore sources sparsely locate on some particular parts, while on the most of common parts, there are few sources. This is why acoustic powers $\mathbf{x}$ become K-sparsity signals when the source plane is discretized into N grids. Such a sparse distribution can be represented by the distribution that has a very high value around the original zero (sparsity) and a long heavy tail (dynamic range of source powers).

Here we apply the Student's-t priors $S t(\boldsymbol{x})$ [7] to enforce the sparsity and wide dynamic range of source power distribution. Owing to the superposition property, hidden variable $\boldsymbol{\gamma}$ is marginalized out for $S t(\boldsymbol{x})=\int p(\boldsymbol{x} \mid \boldsymbol{\gamma}) p(\boldsymbol{\gamma}) d \boldsymbol{\gamma}$, where $p(\boldsymbol{x} \mid \boldsymbol{\gamma})=\mathcal{N}\left(\boldsymbol{x} \mid 0, \boldsymbol{\Sigma}_{x}^{-1}\right)$ is assigned to multivariate Gaussian distribution, in which, $\boldsymbol{\Sigma}_{x}$ denotes power covariance matrix, defined as $\boldsymbol{\Sigma}_{x}=\operatorname{Diag}\{\boldsymbol{\gamma}\}$ with $\boldsymbol{\gamma}=\left\{\gamma_{n}\right\}_{N}$ being the power precision vector; and $p(\gamma)=\prod_{n=1}^{N} \operatorname{Gama}\left(\gamma_{n} \mid a_{\gamma}, b_{\gamma}\right)$, where $a_{\gamma}, b_{\gamma}$ denotes the hyper-parameters of $p(\gamma) .0<\gamma_{n}<1$ greatly promotes the sparsity, while $\gamma_{n} \rightarrow \infty$ makes $S t\left(x_{n}\right)$ to approach Gauss normal distribution, which has no sparsity. Compared to the Double Exponential (DE) prior in [9], $S t\left(x_{n}\right)$ involves different hidden variable $\gamma_{n}$ for every $x_{n}$, while DE prior requires two fix parameters for all $\mathrm{x}$ in order to achieve the same sparsity and heavy tail distribution, as the red curve shown in Fig.1a.

\section{VBA parameter estimations}

In Fig.1a, the graphical model [11] describes the dependencies between the observed data $\mathbf{y}$, unknown variables $\boldsymbol{\theta}=[\mathbf{x}, \boldsymbol{\xi}]^{T}$, their hidden variables $\boldsymbol{\varphi}=[\boldsymbol{\gamma}, \boldsymbol{\nu}]^{T}$ and hyperparameters $\phi=\left[a_{\gamma}, b_{\gamma}, a_{\nu}, b_{\nu}\right]^{T}$. According to Baye's rule, we have $p(\boldsymbol{\theta}, \boldsymbol{\varphi} \mid \boldsymbol{y}) \propto p(\boldsymbol{y} \mid \boldsymbol{\theta}, \boldsymbol{\varphi}) p(\boldsymbol{\theta} \mid \boldsymbol{\varphi}) p(\boldsymbol{\varphi})$. Owing to the multivariate Gaussian likelihood in (2) and the superpositions of Student's-t priors on $\boldsymbol{\theta}$, we specifically obtain the posterior as

$$
\begin{aligned}
p(\boldsymbol{\theta}, \boldsymbol{\varphi} \mid \boldsymbol{y}) & =\mathcal{N}\left(\boldsymbol{x} \mid \boldsymbol{y}-\mathbf{C} \boldsymbol{x}, \boldsymbol{\Sigma}_{\xi}^{-1}\right) \mathcal{N}\left(\boldsymbol{x} \mid 0, \boldsymbol{\Sigma}_{x}^{-1}\right) \\
& \operatorname{Gama}\left(\boldsymbol{\gamma} \mid a_{\gamma}, b_{\gamma}\right) \mathcal{N}\left(\boldsymbol{\xi} \mid 0, \boldsymbol{\Sigma}_{\xi}^{-1}\right) \operatorname{Gama}\left(\boldsymbol{\nu} \mid a_{\nu}, b_{\nu}\right)
\end{aligned}
$$


Owing to the conjugate prior, approximating posterior belongs to Student's-t distribution which consists of multivariate Gaussian $\hat{q}(\boldsymbol{x})$ and Gamma distributions $\hat{q}(\boldsymbol{\gamma}), \hat{q}(\boldsymbol{\nu})$ as:

$$
\left\{\begin{array}{l}
\hat{q}(\boldsymbol{x})=\mathcal{N}\left(\boldsymbol{x} \mid \hat{\boldsymbol{\mu}}_{x}, \hat{\boldsymbol{\Sigma}}_{x}\right) \\
\hat{q}(\boldsymbol{\gamma})=\prod_{n=1}^{N} \operatorname{Gama}\left(\gamma_{n} \mid \hat{a}_{\gamma}, \hat{b}_{\gamma}^{n}\right) \\
\hat{q}(\boldsymbol{\nu})=\prod_{n=1}^{N} \operatorname{Gama}\left(\nu_{n} \mid \hat{a}_{\nu}, \hat{b}_{\nu}^{n}\right),
\end{array}\right.
$$

where $\boldsymbol{x}$ and $\varphi$ are supposed to be mutually independent; and expected variable estimations are as:

$$
\left\{\begin{array}{l}
\hat{\boldsymbol{\mu}}_{x}=\hat{\boldsymbol{\Sigma}}_{x} \mathbf{C}^{T}<\boldsymbol{\Sigma}_{\xi}>\boldsymbol{y} \\
\hat{\boldsymbol{\Sigma}}_{x}=\left(\mathbf{C}^{T}<\boldsymbol{\Sigma}_{\xi}>\mathbf{C}+<\boldsymbol{\Sigma}_{x}>\right)^{-1} \\
\hat{a}_{\gamma}=a_{\gamma}+\frac{N}{2}, \hat{b}_{\gamma}^{n}=b_{\gamma}+\frac{1}{2}<\boldsymbol{x} \boldsymbol{x}^{T}>_{n n} \\
\hat{a}_{\nu}=a_{\nu}+\frac{N}{2}, \hat{b}_{\nu}^{n}=b_{\nu}+\frac{1}{2}<\boldsymbol{\xi} \boldsymbol{\xi}^{T}>_{n n}
\end{array}\right.
$$

where operator $(\cdot)_{n n}$ denotes the $n$th diagonal item, and $\langle\cdot\rangle$ denotes expectation, which are calculated as:

$$
\left\{\begin{array}{l}
<\boldsymbol{\Sigma}_{\xi}>=\operatorname{Diag}\left\{<\nu_{n}>\right\}_{N}=\operatorname{Diag}\left\{<\hat{a}_{\nu} / \hat{b}_{\nu}^{n}>\right\}_{N} \\
<\boldsymbol{\Sigma}_{x}>=\operatorname{Diag}\left\{<\gamma_{n}>\right\}_{N}=\operatorname{Diag}\left\{<\hat{a}_{\gamma} / \hat{b}_{\gamma}^{n}>\right\}_{N} \\
<\boldsymbol{x} \boldsymbol{x}^{T}>=\hat{\boldsymbol{\mu}}_{x} \hat{\boldsymbol{\mu}}_{x}^{T}+\hat{\boldsymbol{\Sigma}}_{x} \\
<\boldsymbol{\xi} \boldsymbol{\xi}^{T}>=\boldsymbol{y} \boldsymbol{y}^{T}-2 \mathbf{C} \hat{\boldsymbol{\mu}}_{x} \boldsymbol{y}^{T}+\mathbf{C}<\boldsymbol{x} \boldsymbol{x}^{T}>\mathbf{C}^{T}
\end{array}\right.
$$

All the solutions in $(5,6)$ require the values of the hyperparameters $\phi=\left[a_{\gamma}, b_{\gamma}, a_{\nu}, b_{\nu}\right]^{T}$. During the iterations of parameter estimations, variables $\boldsymbol{\theta}=[\mathbf{x}, \boldsymbol{\varphi}]$ are firstly computed, then the hyperparameters $\phi$ can be alternatively estimated by making the first partial derivative of variational bound $\mathfrak{L}_{\boldsymbol{\theta}}(\phi)$ equal zero $\left(\frac{\partial \mathfrak{L}}{\partial \phi_{i}}=0\right)$ as follows:

$$
\left\{\begin{array}{l}
\left.\frac{\partial \mathfrak{L}}{\partial a_{\nu}}=N \ln b_{\nu}-N \digamma\left(a_{\nu}\right)+\sum_{n=1}^{N}<\ln \nu_{n}\right\rangle \\
\frac{\partial \mathfrak{L}}{\partial b_{\nu}}=N \frac{a_{\nu}}{b_{\nu}}-\sum_{n=1}^{N}\left\langle\nu_{n}>\right.
\end{array}\right.
$$

where $\left\langle\nu_{n}\right\rangle$ and $\left\langle\gamma_{n}\right\rangle$ are computed in (6), and $\digamma(\cdot)$ denotes the digamma function defined by $\psi(x)=\Gamma^{\prime}(x) / \Gamma(x)$ where $\Gamma(\cdot)$ denotes the Gamma function. $a_{\nu}, b_{\nu}$ can be simultaneously estimated from the same procedure.

\section{Computational analysis}

From the solutions in (5), $\hat{\boldsymbol{\Sigma}}_{x}$ involves the matrix inversion which can not be calculated explicitly nor efficiently. We have to approximate $\hat{\boldsymbol{\Sigma}}_{x}$ with a circulant matrix as $\hat{\boldsymbol{\Sigma}}_{x} \approx\left(<\bar{\nu}>\mathbf{C}^{H} \mathbf{C}+<\bar{\gamma}>\right)^{-1}$, where $\bar{\nu}=\sum_{n=1}^{N} \nu_{n}$, $\bar{\gamma}=\sum_{n=1}^{N} \gamma_{n}$ denote the arithmetic mean. Then the products of circulant matrices can be efficiently computed in the Discrete Fourier Transform (DFT) domain. In (5), the estimated expectation $\hat{\boldsymbol{\mu}}_{x}$ of source powers can be analytically expressed as $\hat{\boldsymbol{\Sigma}}_{x}^{-1} \hat{\boldsymbol{\mu}}_{x}=\mathbf{C}^{H}<\boldsymbol{\Sigma}_{\xi}>\mathbf{y}$. This linear system of equations is solved iteratively with the conjugate gradient algorithm, which requires $O(N \log N)$ computations to treat $\mathrm{N}$ dimension vector $\mathbf{x}$. If $\mathrm{Q}$ iterations are needed, total computations are of $O(Q N \log N)$, which remains moderate burden.

\section{Simulations}

The Simulation configurations are based on the wind tunnel experiments in Fig.3a: the distance between sensor and source plane is $4.50 \mathrm{~m}$; There are $\mathrm{M}=64$ sensors; The source plane is discretized into $5 \mathrm{~cm} \times 5 \mathrm{~cm}$ grids. In Fig. $2 \mathrm{a}$, source powers $\mathrm{x}$ are generated by 4 monopoles and 5 extended sources within $14 \mathrm{~dB}$ dynamic range, and image size are of $27 \times 17$ pixels. The colored noises are generated by using the Gaussian white noises via low pass filter (cut-off frequency $3000 \mathrm{~Hz}$ ), and the averaged Signal-to-Noise Ratio (SNR) is set as low as OdB. (a)

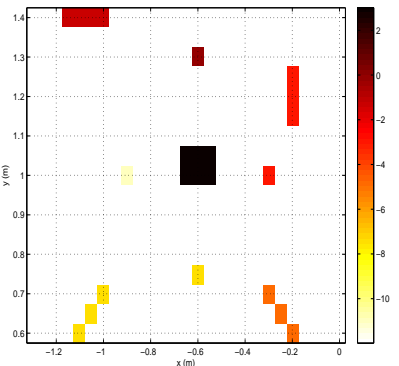

(c)

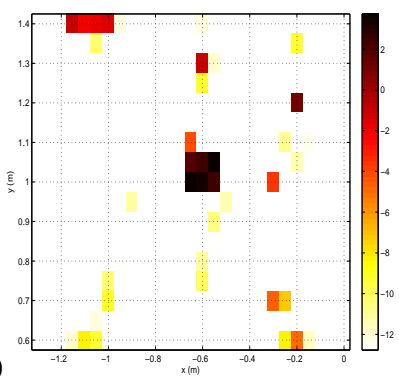

(b)

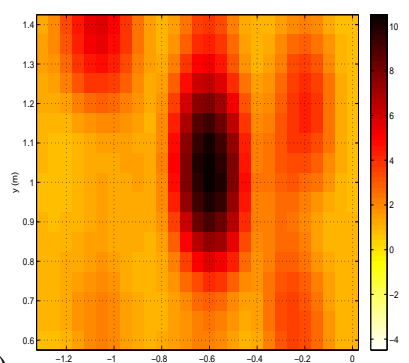

(d)

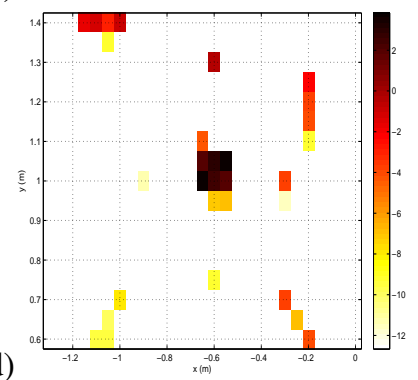

Fig. 2. Simulation at $2500 \mathrm{~Hz}, 0 d B$ SNR in colored noises, $14 d B$ display: (a) Source powers (b) Beamforming powers (c) Bayesian $M A P$ inversion and (d) Proposed VBA inversion

In Fig.2b-d, the beamforming merely gives the strong sources and failed to distinguish most of weak sources; our proposed VBA inference outperforms the MAP method [9] for the more precise localization and power estimations, especially for the better colored noise suppression.

\section{WIND TUNNEL EXPERIMENTS}

Wind tunnel experiments are designed to reconstruct the positions and acoustic powers on the traveling car surface. The grid is of $5 \mathrm{~cm}$, and source plane is thus of $31 \times 101$ pixels. The wind speed is $160 \mathrm{~km} / \mathrm{h}$; there are 524288 samplings with the sampling frequency $f_{s}=2.56 \times 10^{4} \mathrm{~Hz}$. Total samplings are separated into $\mathrm{I}=204$ blocks with 2560 samplings in each bloc. The working frequency is $2500 \mathrm{~Hz}$, which is sensitive to human being. The image results are obtained in frequency domain shown by normalized $\mathrm{dB}$ images with $10 \mathrm{~dB}$ span. Propagation matrix $\mathbf{C}$ in (1) is rectified for the wind refraction and ground reflection respectively as discussed in [9].

Fig.3 illustrates the estimated powers of mentioned methods. Due to the high side-lobe effect, beamforming merely gives a fuzzy image of strong sources in Fig.3b; DAMAS well deconvolves the beamforming image and discovers sources around the wheels and rearview mirror, however, many false targets are also detected on the air in Fig.3c; In Fig.3d and e, MAP inference via DE prior [9] and proposed VBA inference not only manages to distinguish the strong sources around the two wheels and rearview mirror, but also successfully 
(a)

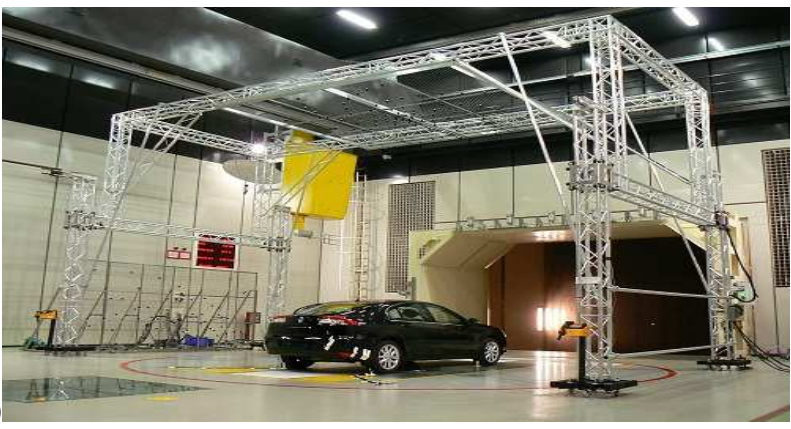

(b)

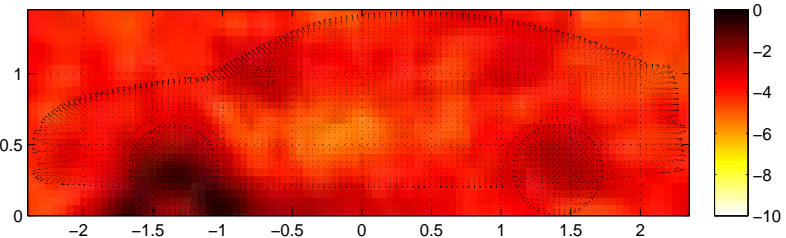

(c)

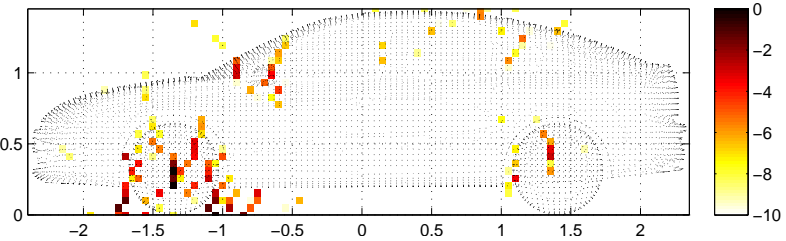

(d)

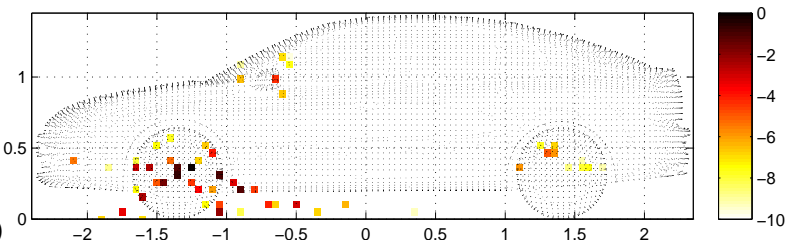

(e)

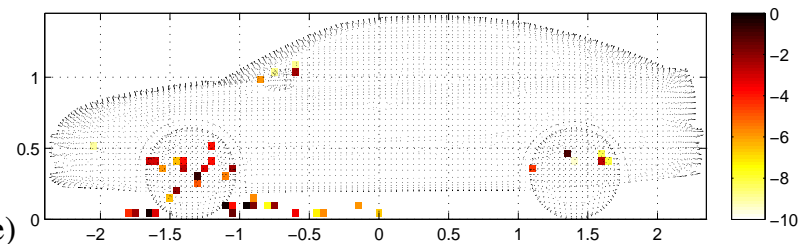

Fig. 3. Vehicle acoustic imaging at 2500Hz: (a) Wind tunnel S2A Renault France (b) Beamforming (c) DAMAS (d) MAP inference and (e) Proposed VBA inference.

reconstructs the week ones on the front cover and light. Furthermore, the background noise suppression in proposed VBA are much better achieved than others thanks to the Student's-t prior on the colored noise.

\section{CONCLUSION}

We proposed a VBA inference via Student's-t priors on source powers and colored noises for super spatial resolution, wide dynamic range and robust parameter estimations. Through simulations and the real data in wind tunnel, proposed approach has been validated comparing to classical methods. But proposed VBA arises computations as $O(Q N \log N)$. For real-time processing, it should be employing the Graphical Processing Unit (GPU) for accelerations.

\section{ACKNOWLEDGMENT}

Great thank to Renault SAS for offering real data.

\section{REFERENCES}

[1] J.C. Chen, K. Yao, and R.E. Hudson, "Source localization and beamforming," Signal Processing Magazine, IEEE, vol. 19, no. 2, pp. 30-39, 2002.

[2] T.F. Brooks and W.M. Humphreys, "A Deconvolution Approach for the Mapping of Acoustic Sources (DAMAS) determined from phased microphone arrays," Journal of Sound and Vibration, vol. 294, no. 4-5, pp. 856-879, 2006.

[3] Tarik Yardibi, Jian Li, Petre Stoica, Nikolas S. Zawodny, and Louis N. Cattafesta, "A covariance fitting approach for correlated acoustic source mapping," Journal of The Acoustical Society of America, vol. 127, no. 5, pp. 2920-31, 2010.

[4] N. Chu, A. Mohammad-Djafari J. Picheral, and N. Gac, "A robust super-resolution approach with sparsity constraint in acoustic imaging," Applied Acoustics, vol. 76, pp. 197-208, 2014.

[5] G. Oliveri, P. Rocca, and A. Massa, "A bayesian-compressive-samplingbased inversion for imaging sparse scatterers," Geoscience and Remote Sensing, IEEE Transactions on, vol. 49, no. 10.

[6] J. Antoni, "A Bayesian approach to sound source reconstruction: optimal basis, regularization, and focusing," The Journal of the Acoustical Society of America, vol. 131, pp. 2873-2890, 2012.

[7] D.G. Tzikas, A.C. Likas, and N.P. Galatsanos, "Variational Bayesian sparse kernel-based blind image deconvolution with Student's-t priors," IEEE Transactions on Image Processing, vol. 18, no. 4, pp. 753-764, 2009.

[8] Ali Mohammad-Djafari, "Bayesian approach with prior models which enforce sparsity in signal and image processing," EURASIP Journal on Advances in Signal Processing, vol. 2012, no. 1, pp. 52, 2012.

[9] N. Chu, A. Mohammad-Djafari, and J. Picheral, "Robust Bayesian super-resolution approach via sparsity enforcing a priori for near-field aeroacoustic source imaging," Journal of Sound and Vibration, vol. 332, no. 18, pp. 4369-4389, 2013.

[10] A. Massa and G. Oliveri, "Bayesian compressive sampling for pattern synthesis with maximally sparse non-uniform linear arrays," IEEE Transactions on Antennas and Propagation, vol. 59, no. 10.

[11] M.I. Jordan, Z. Ghahramani, T.S. Jaakkola, and L.K. Saul, "An introduction to variational methods for graphical models," Machine learning, vol. 37, no. 2, pp. 183-233, 1999. 